\title{
Time uncertainty in quantum gravitational systems
}

\author{
J. Fernando Barbero G., Guillermo A. Mena Marugán, and Eduardo J. S. Villaseñor \\ IMAFF, CSIC, Serrano 113bis-121, 28006 Madrid, Spain
}

(Received 4 August 2003; published 23 February 2004)

\begin{abstract}
It is generally argued that the combined effect of the Heisenberg principle and general relativity leads to a minimum time uncertainty. Most of the analyses supporting this conclusion are based on a perturbative approach to quantization. We consider a simple family of gravitational models, including the Einstein-Rosen waves, in which the (nonlinearized) inclusion of gravity changes the normalization of time translations by a monotonic energy-dependent factor. In these circumstances, it is shown that a maximum time resolution emerges nonperturbatively only if the total energy is bounded. Perturbatively, however, there always exists a minimum uncertainty in the physical time.
\end{abstract}

DOI: 10.1103/PhysRevD.69.044017

PACS number(s): 04.60.Ds, 03.65.Ta, 04.62.+v, 06.30.Ft

Given a quantum state, one can track the passage of time by analyzing the evolution of probability distributions of observables [1]. However, for every observable, there is a characteristic time that places a limit on the ability to detect the evolution. This characteristic time can be estimated as the ratio between the root-mean-square (rms) deviation of the observable and the (absolute value of the) time derivative of its expectation value. In conservative systems, the noncommutativity of quantum mechanics implies that, for all explicitly time-independent observables, this characteristic time is greater or equal than $\hbar / 2$ divided by the rms deviation $\Delta H$ of the energy [1]. As a consequence, any measurement of time has an intrinsic uncertainty $\Delta t$ that satisfies the so-called fourth Heisenberg relation, $\Delta t \Delta H \geqslant \hbar / 2$. For eigenstates of the Hamiltonian, the probability distributions are stationary. To increase the time sensitivity, one must allow for states with a larger and larger energy uncertainty. A perfect time resolution can be reached only when the energy is completely delocalized.

The discussion gets much more involved when general relativity enters the scene. It is commonly accepted that the above quantum mechanical description should be valid in the low-energy regime, or around a background that provides the fundamental state. However, higher-order corrections in this approximation should become important when one considers states with large energy fluctuations, necessary for a good time resolution. Indeed, several arguments indicate that a minimum time structure appears when one includes at least the next-to-leading order contribution to the time uncertainty $[2,3]$.

A way to understand this phenomenon is by the back reaction caused by the energy of the quantum system. In general relativity, this energy curves the spacetime. If the physical time is defined in terms of a unit (asymptotic) timelike Killing vector, the presence of additional energy around the background modifies the normalization of this vector and hence the definition of time. Since this modification depends on the amount of extra energy, quantum uncertainties in the energy give rise to time uncertainties [2]. This mechanism prevents one from attaining the limit of infinite time resolution by increasing the energy fluctuations unless the contributions to the time uncertainty arising from quantum mechanics and general relativity are correlated in a very specific manner.
There exists a certain similarity between these arguments and those supporting the existence of a minimum time (or length) in the string [2-5]. The spacetime uncertainty resulting from scattering processes in string perturbation theory [4] can be understood as produced by the independent fluctuations of the two worldsheet directions [5]. These fluctuations are given by two dual extremal lengths, whose product never vanishes. In fact, the uncertainty in the time direction of the worldsheet is proportional to that in the time-of-flight measurement of the momentum, which increases when one improves the resolution in the spatial direction [5].

This line of reasoning has led to different proposals for the minimum time uncertainty that one should expect in gravitational systems $[3,6,7]$. The simplest proposal is an uncertainty of Planck order [2,3]. Assuming random fluctuations at the Planck scale, an uncertainty that increases with the square root of time has also been suggested [6]. The same behavior was found by Salecker and Wigner (SW) by analyzing a device acting as a clock, with initial position and momentum rms deviations $\Delta x$ and $\Delta p$ and mass $m$ [8]. Again, as time passes, the position uncertainty receives an energy correction $[8,9]$, namely $[\Delta x(t)]^{2}=[\Delta x]^{2}$ $+[t \Delta p / m]^{2}$. The Heisenberg principle implies then that the minimum of the time uncertainty $\Delta t \equiv \Delta x(t)$ (with $c=1$ ) is proportional to $\sqrt{t}[6,8,10]$.

Although these proposals are not free of controversy $[9,11]$, they have originated an increasing interest for the consequences that a minimum time uncertainty of quantum gravitational nature could have in astrophysics $[7,12,13]$. For instance, it has been proposed that this uncertainty might cause a distinctive displacement noise in gravitational wave interferometers $[6,12]$. Another effect would be the loss of phase coherence in the radiation emitted by distant astrophysical sources, which would prevent the formation of diffraction patterns $[13,14]$. This last suggestion has received several criticisms [15].

These predictions are sometimes regarded as observational tests of the time uncertainty in quantum gravity. However, they are deduced in fact under stronger hypotheses, which imply a foamy spacetime. Thus, for gravitational wave detectors a fuzzy concept of distance is assumed [6]. On the other hand, the light coming from extragalactic objects suf- 
fers a significant loss of phase only if there exist spacetime fluctuations which induce random phase variations along the propagation path [14].

The extent to which a minimum time uncertainty leads to testable consequences is obscured by the use of descriptions that are mainly phenomenological, rather than obtained from a consistent quantization in the presence of gravity. Moreover, most of the results that support the existence of a minimum time structure are based on qualitative analyses that involve perturbative corrections [2], but not on complete quantizations of gravitational models. It is hence far from clear whether the conclusions about a minimum uncertainty can be maintained in a nonperturbative quantization. With this motivation in mind, we will study a very specific kind of models whose quantization can be achieved both in a lowenergy or perturbative scheme and by taking gravity into full account.

We consider a dynamical system that, around a certain background or in a certain approximation, can be described by a time-independent Hamiltonian $H_{0}$ with associated time parameter $T$. We assume that this system admits a straightforward quantization. The quantum evolution of any explicitly time-independent observable $A$ is dictated by the Heisenberg equation $i \hbar \partial_{T} A=\left[A, H_{0}\right]$. For simplicity, we also suppose that the spectrum of $H_{0}$ is positive and unbounded, with a nondegenerate fundamental state. At this stage, we let general relativity come into play in a fully nonperturbative way. Our basic hypothesis, inspired by our introductory comments, is that the main effect of plugging in gravity is changing the normalization of the (asymptotic) time translations by an energy-dependent factor. Since the physical time $t$ must be normalized to the unity, we arrive at a relation of the form $t=T V\left(H_{0} E_{P}^{-1}\right)$, where $V$ is a function on $\mathbb{R}^{+}$and $E_{P}$ is a constant energy that can be viewed as a sort of Planck energy for the system.

Since normalization factors are always positive, the function $V$ has to be greater than zero. In addition, to recover $T$ as the time coordinate in the low-energy or perturbative limit in which $H_{0} E_{P}^{-1}$ vanishes, we must have $V(0)=1$. Finally, we introduce the assumptions that $V$ be monotonic and sufficiently smooth to avoid technical complications.

Remarkably, one can construct a consistent quantization of the system in general relativity starting from the quantization that describes the evolution in the time $T$ with Hamiltonian $H_{0}$ [16]. The Hilbert spaces of quantum states on the initial $t=0$ and $T=0$ sections can be identified. Besides, one can check that the evolution in the physical time $t$ is generated (at least classically) by the Hamiltonian $H$ $=E_{P} F\left(H_{0} E_{P}^{-1}\right)$, where the function $F$ is a primitive of $1 / V$. As an operator, $H$ can be defined from $H_{0}$ by means of the spectral theorem. The explicitly time-independent observables satisfy now the equation $i \hbar \partial_{t} A=[A, H]$. We choose $F(0)=0$, so that the ground state energy vanishes also in the presence of gravity. Since $F^{\prime}(x)=1 / V(x)>0$ (because $V$ is positive and smooth), the spectrum of $H$ is hence nonnegative. Apart from a factor $E_{P}$, this spectrum coincides with the image under $F$ of that of $H_{0} E_{P}^{-1}$.
From now on, we will refer to the quantizations with Hamiltonian $H_{0}$ and $H$, respectively, as the perturbative and nonperturbative quantizations. As a motivation for this terminology, note that, since $H=E_{P} F\left(H_{0} E_{P}^{-1}\right)$ and $F(0)=0$, one can think of the perturbative approach as the analysis in the limit $E_{P}^{-1} \rightarrow 0$, in agreement with our previous comments. It can be seen that this analysis reproduces as well the low-energy behavior $H_{0} \approx 0$.

One can doubt that a model of this type may represent a realistic situation in general relativity. However, there is at least one known example: the Einstein-Rosen (ER) waves. These cylindrical gravitational waves are classically equivalent to a massless, axisymmetric scalar field coupled to gravity in three dimensions [17]. In linearized gravity, the corresponding three-dimensional reduction of the metric (in a suitable gauge) is purely Minkowskian, and the dynamics in this Minkowskian time $T$ is generated by the Hamiltonian $H_{0}$ of the free, massless scalar field [16]. Moreover, the time translations $\partial_{T}$ are asymptotically unit even from the perspective of the four-dimensional metric. In cylindrical general relativity without any linearization, on the other hand, the metric in three dimensions is not Minkowskian anymore and the physical time $t$, properly normalized at infinity (both from the three and four-dimensional viewpoints), differs from that of the Minkowski background by a factor that depends on the energy of the free field, $H_{0}$ [16].

Explicitly, $t=T e^{4 G_{3} H_{0}}$ for ER waves, where the inverse energy $G_{3}$ denotes the gravitational constant per unit length in the direction of the axis or, equivalently, the effective Newton constant in three dimensions [16,17]. With our notation, we then have $V(x)=e^{4 x}$ and $E_{P}=1 / G_{3}$. The primitive of $1 / V$ is $F(x)=\left(1-e^{-4 x}\right) / 4$ and the Hamiltonian $H$ in the nonlinear theory is $4 G_{3} H=1-e^{-4 G_{3} H_{0}}$. Thus, the physical energy ranges from zero to $1 /\left(4 G_{3}\right)$.

Let us study the uncertainty in the time $t$ in our family of models. The main observation, already pointed out in the analysis of ER waves [17], is that the physical time $t$ plays the role of evolution parameter in the nonperturbative quantization, whereas this role corresponds to the time $T$ in the perturbative case [16]. In this latter quantization, the physical time $t=T V\left(H_{0} E_{P}^{-1}\right)$ is represented by a one-parameter family of operators. It seems natural to define $V\left(H_{0} E_{P}^{-1}\right)$ in terms of the Hamiltonian $H_{0}$ using the spectral theorem. The operator obtained in this way is positive, because so is the function $V$.

The uncertainty in the nonperturbative quantization is straightforward to analyze. Since the physical time is a dynamical parameter, the fourth Heisenberg relation applies, i.e., $\Delta t \Delta H \geqslant \hbar / 2$. In the light of this relation, we arrive at an unexpected conclusion. Namely, in the description of a fully nonperturbative observer, the existence of a minimum time uncertainty depends only on whether the rms deviation $\Delta H$ of the physical energy is or not bounded from above. Recalling that the spectrum of $H_{0}$ is unbounded and the definition of $H$, one can check that the largest that $\Delta H$ may become is $E_{P} F_{\infty}$. Here, $F_{\infty}$ is the limit of $F(x)$ when $x$ tends to infinity. Therefore, a resolution limit exists in the nonperturbative model if and only if the range of $F$ is bounded. This happens 
to be the case for ER waves, where $F_{\infty}=1 / 4$, and so one has $\Delta t \geqslant 2 \hbar E_{P}^{-1}=2 \hbar G_{3}$. Nevertheless, in more general situations, nothing seems to prevent the range of $F$ to be the whole positive semiaxis. The uncertainty $\Delta t$ might then be decreased to zero by choosing a state with totally uncertain energy $H$.

We now turn to the perturbative approach. Given a quantum state, we can always measure on it the probability distribution of the perturbative energy $H_{0}$, which is stationary because the system is conservative. Via the spectral theorem, this distribution determines that of the operator $V\left(H_{0} E_{P}^{-1}\right)$. We denote by $\Delta V$ the corresponding rms deviation. In order to evaluate the operator $t$, we still need to fix the value of the parameter $T$. As we have explained above, we can detect the passage of the time $T$ in the perturbative framework by examining the evolution of probability distributions of observables in our quantum state. This leads to a statistical measurement of the value of $T$, with a distribution $\rho(T)$ whose uncertainty must be at least of the order of $\Delta T$ $\geqslant \hbar /\left(2 \Delta H_{0}\right)$, according to Heisenberg relation. Note that, in order to capture the intrinsic uncertainties of the system, we choose to evaluate $T$ employing indeed (different copies of) our state vector. Since the described measurements of the perturbative energy $H_{0}$ and $T$ are independent, our measurement procedure assigns to the physical time $t$ a probability distribution which is the product of those found for $T$ and $V$.

Remembering the stationarity of the energy, a straightforward calculation then shows

$$
\begin{aligned}
{[\Delta t]^{2} } & =\int d T \rho(T)\left\langle T^{2} V^{2}-T_{0}^{2}\langle V\rangle^{2}\right\rangle \\
& =T_{0}^{2}[\Delta V]^{2}+\langle V\rangle^{2}[\Delta T]^{2}+[\Delta T \Delta V]^{2} .
\end{aligned}
$$

Here, $T_{0}$ is the mean value of $T$ obtained with the distribution $\rho(T)$, and \langle\rangle denotes expectation value (which can be computed employing the spectral resolution of the identity and the probability distribution of $H_{0}$ ).

The above formula implies that the uncertainty in the physical time cannot vanish in the perturbative quantization. To prove this assertion notice that, in order that $\Delta t$ vanishes, the three factors that appear in Eq. (1) must be zero. But, as soon as $T_{0} \neq 0$, this can only occur if both $\Delta T$ and $\Delta V$ vanish, because $V$ is a positive operator. On the other hand, the spectrum of this operator is, by construction, the image of the spectrum of $H_{0} E_{P}^{-1}$ under the function $V(x)$. With the assumption that this function be monotonic, the vanishing of $\Delta V$ guarantees that the analyzed state is an eigenvector of $H_{0}$. However, owing to the fourth Heisenberg relation, $\Delta T$ may vanish only if the quantum state has an infinite uncertainty in the perturbative energy $H_{0}$. We thus arrive at a contradiction. Hence, the uncertainty in the physical time, as determined by an observer in the perturbative theory, must be strictly positive except perhaps at the initial time of the measurements.

It is instructive to analyze the consequences of Eq. (1) when one keeps only the first perturbative correction to the prediction of ordinary quantum mechanics. Expanding $V\left(H_{0} E_{P}^{-1}\right)$ in powers of $E_{P}^{-1}$ and using $V(0)=1$,

$$
V\left(H_{0} E_{P}^{-1}\right)=1+V^{\prime}(0) H_{0} E_{P}^{-1}+O\left(H_{0} E_{P}^{-1}\right)^{2} .
$$

We then obtain $\langle V\rangle=1$ and $\Delta V=\Delta H_{0} E_{P}^{-1}\left|V^{\prime}(0)\right|$ at leading order. Substituting this in Eq. (1),

$$
[\Delta t]^{2}=\Delta T^{2}+\left[\Delta H_{0}\right]^{2} E_{P}^{-2}\left|V^{\prime}(0)\right|^{2}\left(T_{0}^{2}+\Delta T^{2}\right) .
$$

Remembering that $\Delta T \Delta H_{0} \geqslant \hbar / 2$ and following a line of reasoning similar to that employed to calculate the minimum uncertainty for the SW clock $[6,8]$, one concludes

$$
[\Delta t]^{2} \geqslant \frac{1}{4}\left|V^{\prime}(0)\right|^{2} t_{P}^{2}+\left|V^{\prime}(0)\right| t_{P} T_{0},
$$

where $t_{P}=\hbar E_{P}^{-1}$ can be understood as the Planck time. It is worth pointing out that the deduction of this equation is in fact formally independent of the supposition about the monotonicity of the function $V$.

Formula (4) has a striking resemblance with the kind of effective equation proposed in Ref. [10] to describe the limitation on the measurability of distances. The first term on the right-hand side gives a constant uncertainty of the order of the Planck time $t_{P}$, and can be interpreted as a quantum uncertainty of pure gravitational origin, independent of the details of the state employed in the measurement process [10]. The second contribution is an uncertainty of the order of $\sqrt{t_{P} T_{0}}$, which has the same time dependence that is found in SW devices or in random walk models of Planckian fluctuations $[6,8]$. It can be regarded as originated by the quantum uncertainties that exist on the state used for the time measurements.

We have thus shown that, for the type of models under study, the fact that the physical time is represented as a oneparameter family of operators in the perturbative theory, together with the procedure by which these operators are measured, implies a nonvanishing minimum time uncertainty, lending in this sense confirmation to the perturbatively inspired analyses found in the literature $[2,6]$. On the other hand, in a purely nonperturbative quantization, the physical time can be assigned the role of a dynamical parameter whose uncertainty is restricted only by the standard Heisenberg relation. The time resolution can then be improved without limit if the physical Hamiltonian is unbounded.

Regarding the consequences on gravitational wave detectors and stellar interferometry $[12,13]$, our main remark is that the spacetime structure needs not be foamy in our models. Actually, the uncertainty in the physical time (4) emerges in the perturbative quantization just from two independent processes: the evaluation of $T$ and the measurements of the perturbative energy. In interferometric experiments like those considered here, moreover, an observer in the perturbative theory would register the superposition of two simultaneous signals at the same instant $T$ (which does not even need to be evaluated). Therefore, in our particular class of models, this observer ought not to experiment the kind of phenomena described in Refs. [6,7,12-14]. Although a more detailed analysis and specification of the system is required for definite predictions, we hope that our discussion contributes to emphasize the relevance of the measurement procedure and 
the hypothesis about the foamy behavior of the spacetime (which was explicitly assumed in the above references).

Let us conclude with some general comments. The first one refers to the genuinely nonperturbative results of loop quantum gravity about geometric operators, e.g., the area. The spectrum of these operators is discrete [18], leading to a noncontinuous spacetime picture at small scales. However, these results do not necessarily imply a minimum spacetime resolution. In fact, consecutive area eigenvalues are separated by a (square) distance that vanishes as one approaches the sector of infinite large areas, where an infinite resolution may be reached.

The other comment concerns the feasibility of our models. In Eq. (3) for the time uncertainty, the last term (proportional to $\left.\Delta H_{0}^{2} \Delta T^{2}\right)$ and the first one $\left(\Delta T^{2}\right)$ may be interpreted as contributions with a purely quantum gravitational origin and a standard quantum mechanical origin, respectively [10]. The remaining term should then provide the leading gravitational correction emerging from the uncertainties on the state of the system. One would hence expect that the factor $\left(E_{P}^{-1}\left|V^{\prime}(0)\right| T_{0}\right)^{2}$ multiplying $\Delta H_{0}^{2}$ in this term were independent of the Planck constant $\hbar$, since it must originate from general relativity. As a result, the associated energy $E_{P}$ should be independent of $\hbar$. This is what actually occurs for ER waves, where $E_{P}=1 / G_{3}$. But in more general systems that do not admit reduction to three dimensions, one expects $E_{P}$ to be given by the quantum gravitational Planck scale $\sqrt{\hbar / G}$, where $G$ is (the four-dimensional) Newton constant. In this case, one might argue that the fully nonlinear gravitational behavior will not lead to the simple kind of effects assumed in our models. Even so, the system may possess a scale that replaces $\sqrt{\hbar / G}$ in our discussion and does not vanish when $\hbar=0$. An interesting possibility is the presence of a cosmological constant $\Lambda$. It might then happen that the role of $E_{P}$ could be assigned to $1 / \sqrt{|\Lambda| G^{2}}$ under certain circumstances.

The authors are grateful to A. Ashtekar, M. Varadarajan, and L. Garay for enlightening conversations. This work was supported by the Spanish MCYT projects BFM2002-04031C02 and BFM2001-0213, and E.J.S.V. by a Spanish MEC grant.
[1] A. Galindo and P. Pascual, in Quantum Mechanics I, edited by W. Beiglbock (Springer-Verlag, Berlin, 1990); A. Messiah, Mécanique Quantique I (Dunod, Paris, 1969).

[2] L.J. Garay, Int. J. Mod. Phys. A 10, 145 (1995).

[3] T. Padmanabhan, Class. Quantum Grav. 4, L107 (1987).

[4] D. Amati, M. Ciafoloni, and G. Veneziano, Phys. Lett. B 216, 41 (1989).

[5] R. Guida, K. Konishi, and P. Provero, Mod. Phys. Lett. A 6, 1487 (1991); T. Yoneya, ibid. 4, 1587 (1989).

[6] G. Amelino-Camelia, Phys. Rev. D 62, 024015 (2000).

[7] Y.J. Ng and H. van Dam, Found. Phys. 30, 795 (2000).

[8] H. Salecker and E.P. Wigner, Phys. Rev. 109, 571 (1958).

[9] R.J. Adler, I.M. Nemenman, J.M. Overduin, and D.I. Santiago, Phys. Lett. B 477, 424 (2000); J.C. Baez and S.J. Olson, Class. Quantum Grav. 19, L121 (2002).

[10] G. Amelino-Camelia, Mod. Phys. Lett. A 9, 3415 (1994); 11, 1411 (1996); 13, 1319 (1998).
[11] G. Amelino-Camelia, Phys. Lett. B 477, 436 (2000); Y.J. Ng and H. van Dam, ibid. 477, 429 (2000); Class. Quantum Grav. 20, 393 (2003).

[12] G. Amelino-Camelia, Nature (London) 398, 216 (1999).

[13] R. Lieu and W. Hillman, Astrophys. J. Lett. 585, L77 (2003).

[14] R. Ragazzoni, M. Turatto, and W. Gaessler, Astrophys. J. Lett. 587, L1 (2003).

[15] D.H. Coule, Class. Quantum Grav. 20, 3107 (2003); Y.J. Ng, H. van Dam, and W.A. Christiansen, Astrophys. J. Lett. 591, L87 (2003).

[16] J.F. Barbero G., G.A. Mena Marugán, and E.J.S. Villaseñor, Phys. Rev. D 67, 124006 (2003).

[17] A. Ashtekar and M. Pierri, J. Math. Phys. 37, 6250 (1996); A. Ashtekar, Phys. Rev. Lett. 77, 4864 (1996).

[18] C. Rovelli and L. Smolin, Nucl. Phys. B442, 593 (1995); B456, 753 (1995); A. Ashtekar and J. Lewandowski, Class. Quantum Grav. 14, A55 (1997). 